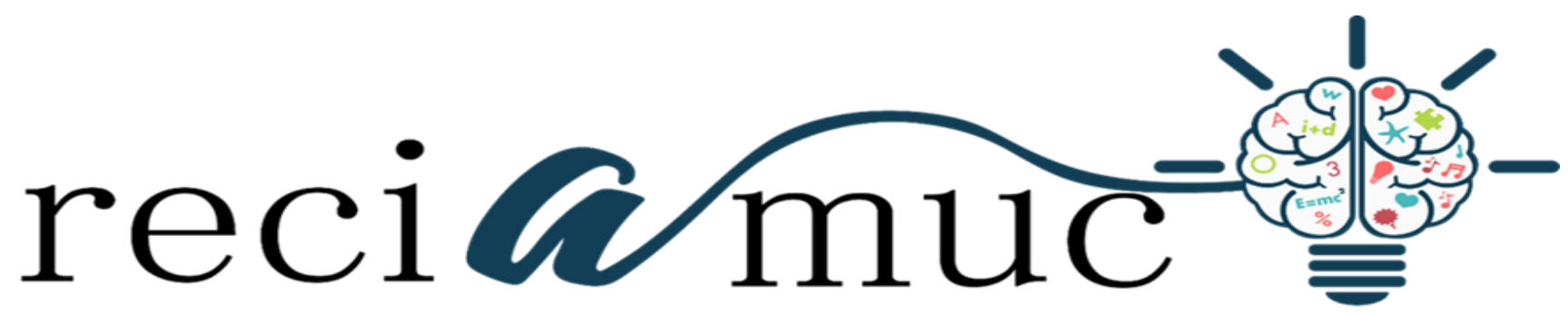

Revista científica de investigación actualización del mundo de las ciencias

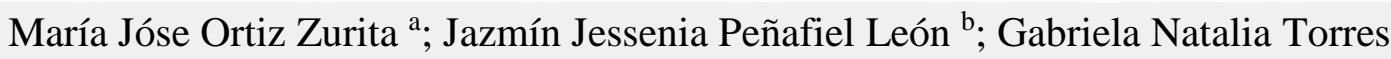
Jara $^{\text {c; }}$ Diana Valeria Delgado Campuzano d; Evelyn Mariuxi Bautista Guaranda ${ }^{\text {e }}$

Propuesta de manejo para el Bosque Protector Cerro Blanco (Ecuador), en base al estudio del área de Conservación Privada Chaparrí (Perú)

Management proposal for Cerro Blanco Protective Forest (Ecuador), based on the study of the Chaparrí Private Conservation area (Perú)

Revista Científica de Investigación actualización del mundo de las Ciencias. Vol. 3 núm., 4, octubre: 2588-0748, 2019, pp. 143-160

DOI: $10.26820 /$ reciamuc/3.(4).octubre.2019.143-160

URL: http://reciamuc.com/index.php/RECIAMUC/article/view/401

Código UNESCO: 5312.90 Economía Sectorial: Turismo

Tipo de Investigación: Artículo de Revisión

(C) RECIAMUC; Editorial Saberes del Conocimiento, 2019

Recibido: 15/05/2019

Aceptado: 07/08/2019

Publicado: 01/10/2019

Correspondencia: mortizz1@unemi.edu.ec

a. Ingeniera en Administración de Empresas Turísticas y Hotelera; Investigador Independiente; Guayaquil, Ecuador; mortizz1@unemi.edu.ec

b. Ingeniera en Administración de Empresas Turísticas y Hotelera; Investigador Independiente; Guayaquil, Ecuador; ipenafiell@unemi.edu.ec

c. Ingeniera en Administración de Empresas Turísticas y Hotelera; Investigador Independiente; Guayaquil, Ecuador; gtorresj@unemi.edu.ec

d. Ingeniera en Ecoturismo; Investigador Independiente; Guayaquil, Ecuador; ddelgadoc@unemi.edu.ec

e. Licenciada en Turismo; Investigador Independiente; Guayaquil, Ecuador; evymar92@ gmail.com 


\section{Propuesta de manejo para el Bosque Protector Cerro Blanco (Ecuador), en base al estudio del área de Conservación Privada Chaparrí (Perú)}

Vol. 3, núm. 4., (2019)

María Jóse Ortiz Zurita; Jazmín Jessenia Peñafiel León; Gabriela Natalia Torres Jara; Diana Valeria Delgado Campuzano; Evelyn Mariuxi Bautista Guaranda

\section{RESUMEN}

Ecuador al igual que el resto de países en vías de desarrollo posee alto potencial para generar productos de naturaleza que atraigan al mercado ecoturístico, el cual día a día se encuentra en búsqueda constante de destinos que les ofrezcan nuevas experiencias. El objetivo fundamental de la presente investigación es plasmar estrategias de manejo para el Bosque Protector Cerro Blanco (Ecuador) a partir de lo planteado por el Área de Conservación Privada Chaparrí (Perú). El diseño de investigación que se llevó a cabo es de tipo una investigación de tipo mixta, por cuanto posee una naturaleza documental y otra de campo. Dentro de las propuestas resultantes, en virtud del trabajo y manejo del área en conjunto con la comunidad del Área de Conservación Privada Chaparrí, se plantea poder establecer alianzas con las comunidades más cercanas. Asimismo, se propuso impulsar circuitos de remo en donde se explique las funcionalidades de este ecotono y la avifauna existente. En conclusión, la actividad turística se encuentra en constante crecimiento y en su mayoría si se le permite el desarrollo de manera sostenible genera beneficios a gran escala.

Palabras claves: Propuesta; Manejo; Bosque; Protector; Blanco. 


\title{
Propuesta de manejo para el Bosque Protector Cerro Blanco (Ecuador), en base al estudio del área de Conservación Privada Chaparrí (Perú)
}

Vol. 3, núm. 4., (2019)

María Jóse Ortiz Zurita; Jazmín Jessenia Peñafiel León; Gabriela Natalia Torres Jara; Diana

Valeria Delgado Campuzano; Evelyn Mariuxi Bautista Guaranda

\begin{abstract}
Ecuador, like the rest of developing countries, has high potential to generate products of nature that attract the ecotourism market, which is constantly searching for destinations that offer new experiences. The main objective of this research is to capture management strategies for the Cerro Blanco Protected Forest (Ecuador) based on what was proposed by the Chaparrí Private Conservation Area (Peru). The research design that was carried out is of a mixed type investigation, because it has a documentary nature and a field nature. Among the resulting proposals, by virtue of the work and management of the area in conjunction with the community of the Chaparrí Private Conservation Area, it is proposed to be able to establish alliances with the closest communities. Likewise, it was proposed to promote rowing circuits explaining the functionalities of this ecotone and the existing birdlife. In conclusion, tourism activity is constantly growing and mostly if sustainable development is allowed, it generates large-scale benefits.
\end{abstract}

Key words: Proposal; Management; Forest; Protector; White. 


\section{Propuesta de manejo para el Bosque Protector Cerro Blanco (Ecuador), en base al estudio del área de Conservación Privada Chaparrí (Perú)}

Vol. 3, núm. 4., (2019)

María Jóse Ortiz Zurita; Jazmín Jessenia Peñafiel León; Gabriela Natalia Torres Jara; Diana Valeria Delgado Campuzano; Evelyn Mariuxi Bautista Guaranda

\section{Introducción.}

El Sector del Ecoturismo es una de las actividades comerciales de mayor crecimiento en el mundo, además de su vital importancia para el sostenimiento de la vida en el planeta.

Una de las primeras definiciones de ecoturismo fue dada por el mexicano Héctor Ceballos 1983 citado en Rhodes, (2015) la cual define al ecoturismo como aquella modalidad del turismo que consiste en viajar a áreas naturales relativamente sin perturbar con el objeto específico de admirar, disfrutar y estudiar su paisaje, su flora y su fauna silvestres, así como las manifestaciones culturales (tanto presentes como pasadas) que allí puedan encontrarse.-

Por otra parte, el concepto según la Organización Mundial de Turismo (2002) menciona ciertas características presentes en esta tipología del turismo: toda forma de turismo basado en la naturaleza en la que la motivación principal de los turistas sea la observación y apreciación de esa naturaleza o de las culturas tradicionales dominantes en las zonas naturales. Incluye elementos educacionales y de interpretación. (Moreno \& Pérez Salazar, 2018)

Existen algunos países en américa latina donde el ecoturismo se encuentra más desarrollado que en otros, ventaja o desarrollo que vale la pena analizar para desarrollar o explotar otros. Para Codling 2000 citado por López (2010) indica que hacer benchmarking es lograr comprender "por qué otros hacen las cosas de manera más eficaz y eficiente”.

De acuerdo a Bogan y English (1994) y Camp (1989), se han identificado los siguientes beneficios del benchmarking: Muestra a las organizaciones como satisfacer las necesidades de los clientes, 2. Identificar las fortalezas y debilidades de la organización, 3. Estimula a la mejora continua.

De acuerdo a los estudios realizados en base al proceso de benchmarking se ha diferenciado dos tipos de análisis, así lo menciona Kozak 2004 citado por Ortiz, I., Ruiz, C. y Molina, E., (2016): 6

Benchmarking en la organización turística y el de destino turístico. 


\section{Propuesta de manejo para el Bosque Protector Cerro Blanco (Ecuador), en base al estudio del área de Conservación Privada Chaparrí (Perú)}

Vol. 3, núm. 4., (2019)

María Jóse Ortiz Zurita; Jazmín Jessenia Peñafiel León; Gabriela Natalia Torres Jara; Diana Valeria Delgado Campuzano; Evelyn Mariuxi Bautista Guaranda

Según la Organización Mundial de Turismo 2005 citado en Vargas, (2009) "En el sector turístico se comienza a percibir conductas de cambio, cuando el visitante solicita servicios que las grandes urbes y el crecimiento industrial les ha privado y sólo el contacto directo con la naturaleza se los puede dar." Ecuador al igual que el resto de países en vías de desarrollo posee alto potencial para generar productos de naturaleza que atraigan a este mercado, el cual día a día se encuentra en búsqueda constante de destinos que les ofrezcan nuevas experiencias. (p. 50)

Partiendo de esta necesidad damos a conocer al Bosque Protector Cerro Blanco el cual se encuentra ubicado en la provincia del Guayas ciudad de Guayaquil, el mismo que ha pasado por varios procesos para poder establecerse como un Bosque Protector.

Dentro de sus antecedentes históricos, podemos mencionar que en 1960 La Cemento Nacional (CN) compró el territorio. En 1989 CN se solicita al Ministerio de Agricultura y Ganadería para la protección de 2.025 hectáreas, parte del territorio fue otorgado en comodatos a la Fundación Natura. En 1990 se creó la infraestructura interpretativa y comenzó a recibir visitantes. Para el año 1992 se crea la Fundación Pro-Bosque (Organización Privada) para la administración del Bosque Protector Cerro Blanco. Posteriormente, en 2000 se extiende a 6.078 hectáreas, y una parte se encuentra en el perímetro urbano. En la actualidad, el Bosque Protector Cerro Blanco pertenece a la entidad privada HOLCIM (antes La CN), la cual es la encargada de financiar su desarrollo.

Actualmente, HOLCIM busca que el Bosque Protector Cerro Blanco pueda auto gestionarse en todas sus operaciones, consideran que el turismo y sus actividades son una buena alternativa ya que compagina perfectamente con el cuidado del entorno natural y el involucramiento de la comunidad.

Considerando la capacidad del Bosque Protector Cerro Blanco para satisfacer la necesidad de los turistas y la búsqueda de su autogestión, surge la necesidad de plantear ideas que puedan ser aplicadas, mismas que deben estar enmarcadas en la "Educación Ambiental" debido a que este es uno de sus principales objetivos y es el marco legal bajo el cual se desarrollan sus actividades. 


\section{Propuesta de manejo para el Bosque Protector Cerro Blanco (Ecuador), en base al estudio del área de Conservación Privada Chaparrí (Perú)}

Vol. 3, núm. 4., (2019)

María Jóse Ortiz Zurita; Jazmín Jessenia Peñafiel León; Gabriela Natalia Torres Jara; Diana Valeria Delgado Campuzano; Evelyn Mariuxi Bautista Guaranda

La presente investigación se lleva a cabo con la finalidad de plasmar una estrategia de manejo para el Bosque Protector Cerro Blanco (Ecuador) a partir de lo planteado por el Área de Conservación Privada Chaparrí (Perú).

\section{Materiales y Métodos.}

El presente estudio se clasificó como una investigación de tipo mixta, por cuanto posee una naturaleza documental y otra de campo.

En cuanto a la investigación documental se llevó a cabo la recolección de información por medio de las páginas webs oficiales del Bosque Protector Cerro Blanco, además de documentación facilitada por esta entidad.

Con relación a la investigación de campo, la técnica principal aplicada fue la entrevista, la cual se aplicó al personal del área en estudio con los que se sostuvieron conversaciones profesionales acerca del objeto de estudio.

El estudio se llevó a cabo en el Bosque Protector Cerro Blanco, Ecuador y área de Conservación Privada Chaparrí, de Perú.

En cuanto al análisis cualitativo se llevó a cabo a partir de la recolección estructurada de la información por medio de entrevistas abiertas al personal que labora en la mencionada área. Por otra parte, el análisis cualitativo se llevó a cabo a partir de la información recolectada de las páginas web oficiales del área en estudio y de la documentación proveída por la administración de la misma, la cual se tradujo posteriormente en datos estadísticos (porcentajes) para la comparación de la variable y la elaboración de la propuesta.

Las variables en estudio fueron las características del Bosque Protector Cerro Blanco vs las características del área de Conservación Privada Chaparrí. Asimismo, en el ámbito Administrativo se estudiaron personal responsable, manejo de las áreas, objetivos propuestos, beneficios obtenidos, producto Estrella, mercado y promoción, de ambos destinos. 


\section{Propuesta de manejo para el Bosque Protector Cerro Blanco (Ecuador), en base al estudio del área de Conservación Privada Chaparrí (Perú)}

Vol. 3, núm. 4., (2019)

María Jóse Ortiz Zurita; Jazmín Jessenia Peñafiel León; Gabriela Natalia Torres Jara; Diana Valeria Delgado Campuzano; Evelyn Mariuxi Bautista Guaranda

\section{Resultados.}

Bosque Protector Cerro Blanco

\section{Características}

Cerro Blanco es un bosque seco tropical, en donde las temperaturas llegan a ser mayores o iguales a $24^{\circ}-26^{\circ} \mathrm{C}$ con una precipitación anual entre 800 a $1200 \mathrm{~mm}$. Se ve influenciado por la corriente marina de Humboltd que bordea las costas del Pacífico que vienen desde el Sur hacia el Norte causando desertificación en zonas continentales cercanas por su naturaleza fría. (Ayón, 2013)

A toda esta área como ya se ha mencionado se la conoce como Eco-región Tumbesina en la cual está ubicada el Bosque Protector Cerro Blanco, designada cono área de importancia para las aves entre 1999 y 2000. (Santander, Freile, \& Loor-Vela, 2009)

\section{Acciones tomadas}

Siguiendo su Misión propuesta de apoyar a la conservación de la biodiversidad del bosque seco tropical de la costa ecuatoriana, han realizado una gran variedad de proyectos como iniciativa propia o en conjunto con organizaciones, entre las cuáles mencionamos las siguientes: Proceso de adaptación de 7 papagayos desde reproducidos en cautiverio para su posterior liberación, curso de Intérpretes Ambientales, 1er. Eco-Festival: 3400 visitante, 1 ir. Curso - taller de Aves del Bosque Cerro Blanco, 1era. Expedición Chester Zoo: 11 técnicos del Zoológico de Inglaterra visitaron el Bosque en el marco de un proyecto de conservación de la Lora Amazonas, entre otras.

\section{Oferta turística actual}

El ingreso al Bosque Protector Cerro Blanco tiene los siguientes costos: adultos $\$ 4$, niños $\$ 3$ y personas de la tercera edad $\$ 2$. De acuerdo a la oferta que poseen enlistamos sus productos: senderismo, guía para observación de aves, campamentos y otros atractivos y servicios tales como área infantil, área de descanso, área de meditación, área de picnic, mirador Buena Vista - glorieta con hamacas y charlas sobre temas de conservación con técnicos especializados. 


\section{Propuesta de manejo para el Bosque Protector Cerro Blanco (Ecuador), en base al estudio del área de Conservación Privada Chaparrí (Perú)}

Vol. 3, núm. 4., (2019)

María Jóse Ortiz Zurita; Jazmín Jessenia Peñafiel León; Gabriela Natalia Torres Jara; Diana Valeria Delgado Campuzano; Evelyn Mariuxi Bautista Guaranda

\section{Análisis del mercado}

El Bosque Protector Cerro Blanco tiene marcada dos temporadas entre las cuales la época seca dura alrededor de 6 meses. De manera general el número de visitas que tuvo el Bosque Protector Cerro Blanco durante el 2017 aumentó el 1.15\% con respecto al año 2016.

\section{Recursos}

De acuerdo a los recursos que posee el bosque mencionamos que tienen contabilizados alrededor de 180 aves, 40 especies de mamíferos y 30 especies correspondientes a herpetofauna.

\section{Análisis Administrativo}

Lo que se busca analizando este aspecto dentro de las áreas seleccionadas es conocer la estructura organizacional y sus niveles de mando, con la finalidad de poder conocer las fortalezas o debilidades de las mismas.

\section{Organigrama}

Dentro del Bosque Protector Cerro Blanco el organigrama administrativo cuenta en primer nivel con un Director Ejecutivo; en segundo nivel el Contador Administrativo y el Asistente del Director; en el tercer nivel se despliegan áreas de Guardaparques, Centro de rescate, Viveros y proyectos, Contabilidad, Turismo, Mantenimiento y Guardianía.

\section{Objetivos planteados}

- Concienciar a los habitantes de las comunas cercanas al Bosque Protector Cerro Blanco sobre la importancia de la biodiversidad para la conservación del Bosque seco tropical.

- Promover el cambio de actitud de los habitantes para que emprendan un papel activo en la conservación del ecosistema bosque seco.

- Generar la adopción de temas de conservación del bosque seco en los currículos de los centros educativos de las comunas cercanas al Bosque Protector Cerro Blanco. 


\section{Propuesta de manejo para el Bosque Protector Cerro Blanco (Ecuador), en base al estudio del área de Conservación Privada Chaparrí (Perú)}

Vol. 3, núm. 4., (2019)

María Jóse Ortiz Zurita; Jazmín Jessenia Peñafiel León; Gabriela Natalia Torres Jara; Diana Valeria Delgado Campuzano; Evelyn Mariuxi Bautista Guaranda

\section{Proceso Interno del manejo turístico}

El turista busca información del Bosque Protector Cerro Blanco a través de Guías de turismo, Internet, website, Facebook, twitter e Instagram y se pone en contacto por medio de correo, whatsapp o messenger para pedir más información o hacer una reservación.

Las reservas se registran en la "Agenda de Reservas" con la fecha y hora indicada por el visitante, sendero elegido, se asigna un número de guías según la cantidad de visitantes, se consulta si el guía lo requiere en español o inglés y se pide al visitante hacer un pago de un anticipo para confirmar la reserva.

- Se contacta a los guías y se les informa de la reserva detallándoles el tipo de visitante y tipo de grupo (estudiantes, familia, amigos, etc.)

- Se coordina con la persona a cargo del mantenimiento para verificar las condiciones de limpieza de baños, centro de visitantes, etc.

- Se informa al guardia de la puerta principal sobre la reservación, guías asignados, etc.

- El día anterior se envía un recordatorio a los guías haciendo hincapié en llegar temprano.

- El día de la visita los guías deben llegar unos 10 a 15 minutos antes del grupo, bien uniformados, verifican las condiciones de las instalaciones y prepararse para recibir al grupo.

- Cuando el grupo llega los guías los reciben, dan la bienvenida, la presentación, breve charla de introducción, una dinámica grupal, división de grupos e ingreso al sendero.

\section{Beneficios de la Comunidad}

El beneficio que actualmente recibe la comunidad de la actividad desarrollada por el Bosque Protector Cerro Blanco se refleja en una mejor calidad del aire, una nueva opción de atracción, barrera natural contra viento y desastres naturales.

Existen beneficiarios directos de la comunidad debido a que muchos de sus colaboradores son personas que viven en las comunidades cercanas, asimismo si se necesita servicios 


\section{Propuesta de manejo para el Bosque Protector Cerro Blanco (Ecuador), en base al estudio del área de Conservación Privada Chaparrí (Perú)}

Vol. 3, núm. 4., (2019)

María Jóse Ortiz Zurita; Jazmín Jessenia Peñafiel León; Gabriela Natalia Torres Jara; Diana Valeria Delgado Campuzano; Evelyn Mariuxi Bautista Guaranda

complementarios para los turistas como alimentación, hospedaje, transporte se hace uso de los que son ofrecidos por la comunidad.

\section{Producto Estrella}

No cuentan con un producto estrella, pero el sendero "Mono Aullador" es el que más acogida está presentando actualmente.

\section{Mercado}

Considerando que el propósito es la Educación Ambiental, el mercado meta ha sido los estudiantes de todos los niveles. En cuanto a turismo extranjero tienen afluencia principalmente de Alemania, Estados Unidos, Canadá e Inglaterra.

\section{Promoción}

La evolución del turismo se ha visto marcado por el avance de las comunicaciones y la tecnología, es por esto que el Bosque Protector Cerro Blanco busca estar presente en las redes sociales como Faceboook, Twitter, Instagram manteniendo la interacción con el público en general.

De manera más específica y considerando el mercado al que están enfocados han elaborado un "Programa promocional de visitas" aún no ejecutado que se encuentra orientado a Visitar escuelas y colegios para promocionar las visitas educativas al bosque.

\section{Resultado General}

De manera general se concluye que la actividad turística les genera alrededor de $\$ 18.000$ al año y reciben alrededor de 5.000 visitantes al año.

Área de Conservación Privada Chaparrí

Características 


\section{Propuesta de manejo para el Bosque Protector Cerro Blanco (Ecuador), en base al estudio del área de Conservación Privada Chaparrí (Perú)}

Vol. 3, núm. 4., (2019)

María Jóse Ortiz Zurita; Jazmín Jessenia Peñafiel León; Gabriela Natalia Torres Jara; Diana Valeria Delgado Campuzano; Evelyn Mariuxi Bautista Guaranda

El Área de Conservación Privada Chaparrí se encuentra ubicada en el límite sur de la ecorregión denominada Bosque Seco ecuatorial, su ubicación es considerada estratégica debido a que tiene cercanía con áreas protegidas por el estado como el Santuario Histórico Bosque de Pomac y la zona reservada de Laquipampa, de esta manera Chaparrí sirve como un corredor biológico entre estas dos áreas. Tiene una extensión de 34.312 h. y se ubica dentro de territorio comunitario "Santa Catalina de Chongoyape" cuya extensión es de 41.260h en la Provincia de Chiclayo, Departamento de Lambayaque.

Esta reserva tuvo origen gracias a que las comunidades campesinas recibieron grandes extensiones de terreno después de la reforma agraria por la que pasó Perú en el año 69, la propuesta para que sea un Área de Conservación Privada fue dada por el Sr. Heinz Plenge, actual director de "La Huerta de Chaparri”, así se inició el trabajo en conjunto con la comunidad para constituirse como Área de Conservación firmando convenios y estableciendo los objetivos que se querían lograr, entre ellos el principal era ofertar un producto turístico considerando que es una actividad que permite incorporar la conservación tanto del entorno como de las especies.

\section{Acciones tomadas}

El Área de Conservación Privada Chaparrí se encuentra muy apegada a la conservación de especies propias del sector entre sus proyectos de conservación podemos mencionar los siguientes: centro de rescate y rehabilitación para osos Andinos, siendo la única en todo Perú, reintroducción de la pava aliblanca lo que ha ayudado a que actualmente se repueblen naturalmente, reintroducción del guanaco camélido más grande de Sudamérica, recuperación del Cóndor Andino, se encuentran trabajando en la introducción de nuevos ejemplares y en un programa de oferta de alimento suplementario.

\section{Oferta turística actual}

Para poder ingresar al Área de Conservación Privada Chaparrí se debe hacer el pago de \$3,06 si solo se trata de un full day, si es el caso de pernoctación el valor es de $\$ 9,16$ lo cual corresponde al permiso por ingreso. 


\section{Propuesta de manejo para el Bosque Protector Cerro Blanco (Ecuador), en base al estudio del área de Conservación Privada Chaparrí (Perú)}

Vol. 3, núm. 4., (2019)

María Jóse Ortiz Zurita; Jazmín Jessenia Peñafiel León; Gabriela Natalia Torres Jara; Diana Valeria Delgado Campuzano; Evelyn Mariuxi Bautista Guaranda

Como la reserva es manejada por la comunidad muchos de ellos han empezado con emprendimientos que sirven como actividades complementarias a la oferta de turismo como cuatrimotos para realizar recorridos en áreas cercanas a la población, otro comunero está implementando áreas de camping, entre otros.

Cuentan con 6 habitaciones estándar, 5 superiores, 2 suite con baño privado, 1 habitación doble y 3 individuales con baño compartido, los siguientes precios incluyen guía local por 1 día y 3 comidas.

\section{Análisis del mercado}

La Reserva Chaparrí tiene temporadas altas que van de mayo a septiembre y temporadas bajas en los meses de Junio - Agosto y Enero - Abril. De manera general la reserva ha presentado un ligero incremento de turistas extranjeros y decrecimiento en el mercado nacional, en cuanto al primer mercado se debe a que han estado presentes esporádicamente en ferias como la de Rutland en Inglaterra, y en cuanto a su mercado nacional principalmente viene por parte de colegios, universidades, delegaciones de institutos, empresas, entre otros que generalmente realizan visitas de un día.

\section{Recursos}

Entre las especies presentes dentro del Área se encuentran registradas 65 aves y 9 especies de mamíferos de las cuales 21 y 9 especies están consideradas en peligro a nivel mundial respectivamente, además hasta el 60\% de anfibios y reptiles de esta región son endémicos.

\section{Análisis Administrativo}

\section{Organigrama}

El Área de Conservación Privada Chaparrí se encuentra manejada sobre la siguiente estructura organizacional: Presidente: Presidente de la Comunidad, Vicepresidente: Propietario de la Huerta Encantada, Secretario: Representante de las Juntas de Administración Local, Tesorero: Un 


\section{Propuesta de manejo para el Bosque Protector Cerro Blanco (Ecuador), en base al estudio del área de Conservación Privada Chaparrí (Perú)}

Vol. 3, núm. 4., (2019)

María Jóse Ortiz Zurita; Jazmín Jessenia Peñafiel León; Gabriela Natalia Torres Jara; Diana

Valeria Delgado Campuzano; Evelyn Mariuxi Bautista Guaranda

representante de guías, Fiscal: Representante de las rondas campesinas, Vocales: Director de la Reserva Ecológica Chaparrí, Representante del Comité de Gestión, Representante de las recaudadoras.

\section{Objetivos planteados}

De manera puntual tienen planteados objetivos específicos relacionados a los pilares fundamentales del ecoturismo como los siguientes: Desarrollar el concepto de que las comunidades deben crear sus propios proyectos que les permita el desarrollo sostenible en el tiempo sin una ayuda permanente de mano extranjera, Preservar el ecosistema y hacer uso responsable del mismo para el desarrollo de actividades económicas que permita generar ingresos y mejorar la calidad de vida de los comuneros, Informar al público en general de las actividades que se han desarrollado, se desarrollan y se plantean desarrollar dentro de la Reserva.

\section{Proceso Interno del manejo turístico}

Los turistas tanto nacionales como extranjeros se informan del lugar por medio de la página web, redes sociales y formulan la solicitud de visita por vía telefónica o correo electrónico, la cual va directo a una base operadora que es la encargada de organizar el calendario de visitas y coordinar en conjunto con la Estación de Guías Chongoyape y el alojamiento por medio de Chaparrí Expeditions.

\section{Beneficios de la Comunidad}

El turismo dentro de la comunidad y el trabajo en conjunto ha podido aportar a la económica de los comuneros e inclusive a personas externas, es decir se ha obtenido beneficios tanto directos como indirectos, la cantidad aproximada de estos beneficiarios es de 3.000 personas, y como se ha mencionado anteriormente el ingreso que se genera se divide por porcentajes. 


\section{Propuesta de manejo para el Bosque Protector Cerro Blanco (Ecuador), en base al estudio del área de Conservación Privada Chaparrí (Perú)}

Vol. 3, núm. 4., (2019)

María Jóse Ortiz Zurita; Jazmín Jessenia Peñafiel León; Gabriela Natalia Torres Jara; Diana Valeria Delgado Campuzano; Evelyn Mariuxi Bautista Guaranda

\section{Producto Estrella}

El turismo arqueológico es el mejor consolidado en el Perú, sin embargo, el turismo gastronómico, sol y playa, aviturismo, entre otros, son segmentos que se encuentran abriendo mercado. En el caso del Área de Conservación Privada Chaparrí apuntan a un mercado específico como es el avistamiento de aves, el cuál es considerado su producto estrella.

\section{Mercado}

Para su producto "avistamiento de aves", sus principales mercados provienen de Estados Unidos, Inglaterra, Francia y Alemania. Dentro del mercado nacional destacan principalmente centros educativos.

\section{Promoción}

Últimamente no han realizado promoción turística, debido a que han centralizado esfuerzos y recursos económicos a problemas presentes como invasiones.

\section{Resultados Generales}

De manera general se concluye que la actividad turística les genera alrededor de $\$ 20.000$ al año solo por pago de permiso de entrada y reciben alrededor de 8.000 visitantes al año.

\section{Propuesta}

Ya habiendo considerado todos los aspectos mencionados anteriormente presentamos a continuación las propuestas desarrolladas:

1. Uno de los puntos importantes que hay que resaltar del Área de Conservación Privada Chaparrí es su trabajo y manejo del área en conjunto con la comunidad, es por esto que se plantea en su principio poder establecer alianzas con las comunidades más cercanas, con la finalidad de desarrollar actividades que los involucren teniendo presente la concienciación ambiental, por 


\section{Propuesta de manejo para el Bosque Protector Cerro Blanco (Ecuador), en base al estudio del área de Conservación Privada Chaparrí (Perú)}

Vol. 3, núm. 4., (2019)

María Jóse Ortiz Zurita; Jazmín Jessenia Peñafiel León; Gabriela Natalia Torres Jara; Diana Valeria Delgado Campuzano; Evelyn Mariuxi Bautista Guaranda

ejemplo: proponer talleres para elaborar artesanías con material reciclado, semillas o materiales propios del sector, las mismas que después pueden ser vendidas a los visitantes.

2. Utilizando el mismo entorno de la comunidad se propone impulsar circuitos de remo en donde se explique las funcionalidades de este ecotono y la avifauna existente. Las capacitaciones en cuanto al servicio del cliente, manejo de negocios, etc. puede ser dada directamente por el Ministerio de Turismo.

3. Como algo sucesivo al primer punto se propone establecer un circuito combinado de avistamiento de aves entre el Bosque Protector Cerro Blanco y la comunidad Puerto Hondo, dando a conocer las diferentes especies existentes en estos dos diferentes tipos de entornos.

Se plantea establecer una ruta de ciclismo dentro del Bosque Protector Cerro Blanco, para diversificar la oferta existente, a su vez se propone conectar con la comunidad de Puerto Hondo haciendo uso del ciclo vía ya establecida en la carretera principal, incentivando la gastronomía local, misma que en tiempo atrás logró fomentar el lugar como una parada obligatoria para las personas que se dirigen a las playas. La implementación o adecuación de esta ruta dentro del bosque, no debería generar gastos mayores a más de señaléticas, la implementación de dispensadores de agua, y botiquín de primeros auxilios, debido a que ya existe elementos educacionales y de interpretación.

4. De acuerdo a los resultados que ha experimentado el Área de Conservación Privada Chaparrí al apuntar a un nicho de mercado objetivo como es el avistamiento de aves, y considerando que el Bosque Protector Cerro Blanco posee fauna de gran importancia, se propone ampliar la visión hacia este mercado tomando en cuenta que han tenido una reducción del mercado "estudiantes" y que este nuevo nicho está en crecimiento, así como indica la Fundación Mindo Cloudforest 2006, citado por (Vargas J. D., 2008)

El mercado es creciente ya que quienes practican esta actividad tienden a sentir la necesidad y el deseo de seguir colectando más y más especies, hasta el punto que se podría decir que actualmente 


\section{Propuesta de manejo para el Bosque Protector Cerro Blanco (Ecuador), en base al estudio del área de Conservación Privada Chaparrí (Perú)}

Vol. 3, núm. 4., (2019)

María Jóse Ortiz Zurita; Jazmín Jessenia Peñafiel León; Gabriela Natalia Torres Jara; Diana Valeria Delgado Campuzano; Evelyn Mariuxi Bautista Guaranda

la observación de aves se ha convertido, para millones de personas, más que en un deporte o pasatiempo, es una pasión.

A su vez indican que este mercado representa grandes ingresos económicos, solo en EE.UU. se generó alrededor de 36.000.000.000 de dólares, con un número aproximado de 48.000.000 observadores de aves, quienes gastaron esa cantidad en sus viajes y en equipo únicamente para observar y fotografiar aves silvestres.

5. Esta propuesta va de la mano con la implementación de alojamiento debido a que se facilitaría la estadía para los birdwatchers, que de acuerdo a varios conocedores la mejor hora de avistamiento es en las mañanas donde aún hay ausencia de ruido.

Es necesario mencionar que esta propuesta también se apega a la Educación Ambiental debido a que mientras más avistamientos se realicen se nutren los listados de aves actuales, inclusive este mercado es conocedor y bien informado por lo que podrían aportar conocimientos de una u otra manera.

6. Presentar propuestas de visitas especializadas a las Agencias de Viajes cuyo perfil del cliente sea enfocado en realizar un turismo específico, como la observación de aves, murciélagos, entre otros.

7. Se propone que el Bosque Protector Cerro Blanco tenga a la vista souvenirs para que los visitantes puedan tener el incentivo de gasto, los mismos que deben estar relacionados con el bosque, como camisetas, bolígrafos, mapas, llaveros, cuadernillos informativos, etc.

\section{Conclusiones.}

Considero que el turismo en países Latinoamericanos se está viendo más atractivo en comparación a países europeos o de Medio Oriente, los que actualmente se encuentran con problemas de inseguridad, aspecto considerado primordial al momento de realizar un viaje. 


\section{Propuesta de manejo para el Bosque Protector Cerro Blanco (Ecuador), en base al estudio del área de Conservación Privada Chaparrí (Perú)}

Vol. 3, núm. 4., (2019)

María Jóse Ortiz Zurita; Jazmín Jessenia Peñafiel León; Gabriela Natalia Torres Jara; Diana Valeria Delgado Campuzano; Evelyn Mariuxi Bautista Guaranda

En base a la experiencia también se reconoció que no existe una real competencia en el mercado de "aviturismo", lo ideal sería crear corredores biológicos en donde los turistas ornitólogos que generalmente cuentan con 10 a 12 días disponibles, puedan recorrer la mayor cantidad de áreas para acaparar diversidad de aves.

Considero que este nicho de mercado atribuye, sin duda alguna, conocimiento tanto para las áreas involucradas como a la sociedad en general ya que como se ha mencionado la mayoría de estos turistas son personas con vastos conocimientos.

El Bosque Protector Cerro Blanco al estar enmarcado en la Educación Ambiental, al tener el enfoque de mercado a los centros educativos y estos a su vez al no tener el permiso de salida, genera un problema para el buen desarrollo de la actividad del bosque, es por esto que se propone tratar de atraer a otro mercado orientando las actividades en el aporte de conocimiento, en la concienciación del entorno y la protección del recurso.

Como se conoce el turismo no es la panacea para resolver los problemas actuales, sin embargo, como también es de conocimiento general está actividad se encuentra en constante crecimiento, y en su mayoría si se le permite el desarrollo de manera sostenible genera beneficios a gran escala.

\section{Bibliografía.}

Ayón, J. (2013). XV Curso de intérpretes Ambientales del Bosque Protector Cerro Blanco. [Apuntes Académicos] Fundación Pro.Bosque. (E. Bautista, Entrevistador)

Bogan, \& English. (1994). Benchmarking for Best Practices: Winning through Innovative Adaptation. NewYork: McGraw-Hill.

Camp, R. (1989). Benchmarking: The Search for Industry Best Practices that Leads to Superior Performance. Milwaukee: American Society for Quality Control, Quality Press.

Lopez, L. (2010). Benchmarking y su Aplicación en Turismo. Revista de Estudos Politécnicos Polytechnical Studies Review, 8(14), 163-180. Obtenido de http://www.scielo.mec.pt/pdf/tek/n14/n14a12.pdf

Moreno, D., \& Pérez Salazar, J. (2018). Propuesta de creación de un área protegida municipal de recreación en el sector las pozas del cantón Milagro. RECIMUNDO, 2(2), 148-182. 


\section{Propuesta de manejo para el Bosque Protector Cerro Blanco (Ecuador), en base al estudio del área de Conservación Privada Chaparrí (Perú)}

Vol. 3, núm. 4., (2019)

María Jóse Ortiz Zurita; Jazmín Jessenia Peñafiel León; Gabriela Natalia Torres Jara; Diana Valeria Delgado Campuzano; Evelyn Mariuxi Bautista Guaranda

Organización Mundial del Turismo. (2002). Organización Mundial del Turismo. Obtenido de http://sdt.unwto.org/es/content/ecoturismo-y-areas-protegidas

Ortiz, I., Ruiz, C., \& Molina, E. (2016). El Benchmarking aplicado al Turismo Idiomático: Una herramienta para lograr estrategias innovadoras de gestión.Caso de York (Inglaterra) y Córdoba (España). International Journal of Scientific Managment Tourism, 12, 267-289. Obtenido de https://dialnet.unirioja.es/servlet/articulo?codigo=5665938

Rhodes, A. (04 de Febrero de 2015). Ecoturismo Genuino. Obtenido de http://www.ecoturismogenuino.com/inicio/2015/02/04/definiciones-ecoturismo/

Santander, T., Freile, J., \& Loor-Vela, S. (2009). Important Bird Areas Americas - Priority sites for biodiversity conservation. En C. D. Devenish, R. Clay, I. Davidson, I. YÉPEZ, \& B. Internacional (Ed.), Important Bird Areas (Vol. BirdLife Conservation Series No. 16, págs. 187-196). Quito, Ecuador: Poligráfica C.A. .

Vargas, J. D. (2008). Comercializando nuestras aves; el aviturismo como estrategia de conservación en Costa Rica. Avis, 8, 3-9. Obtenido de http://www.uniondeornitologos.com/wpcontent/uploads/AVIS/8/AVIS\%208\%202\%20Aviturismo.pdf

Vargas, U. G. (2009). Turismo y espacios naturales protegidos en Costa Rica Enfrentamiento o Concertación. Ciencias Sociales, 49-78. Obtenido de https://revistacienciassociales.ucr.ac.cr/images/revistas/RCS123_124/04VARGAS.pdf

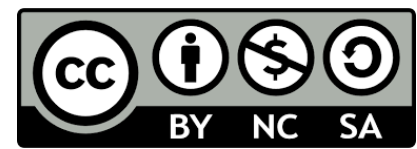

RECONOCIMIENTO-NOCOMERCIAL-COMPARTIRIGUAL

CC BY-NC-SA

ESTA LICENCIA PERMITE A OTROS ENTREMEZCLAR, AJUSTAR Y CONSTRUIR A PARTIR DE SU OBRA CON FINES NO COMERCIALES, SIEMPRE Y CUANDO LE RECONOZCAN LA AUTORÍA Y SUS NUEVAS CREACIONES ESTÉN BAJO UNA LICENCIA CON LOS MISMOS TÉRMINOS. 